\title{
Explaining the isotope effect on heat transport in L-mode with the collisional electron-ion energy exchange
}

P. A. Schneider ${ }^{1}$, A. Bustos ${ }^{2}$, P. Hennequin ${ }^{3}$, F. Ryter ${ }^{1}$, M. Bernert ${ }^{1}$, M. Cavedon ${ }^{1}$, M. G. Dunne $^{1}$, R. Fischer ${ }^{1}$, T. Görler ${ }^{1}$, T. Happel ${ }^{1}$, V. Igochine ${ }^{1}$, B. Kurzan ${ }^{1}$, A. Lebschy ${ }^{1}$, R. M. McDermott $^{1}$, P. Morel ${ }^{3}$, M. Willensdorfer ${ }^{1}$, the ASDEX Upgrade Team ${ }^{1}$ and the EUROfusion MST1 Team*

${ }^{1}$ Max-Planck-Institut für Plasmaphysik, Boltzmannstr. 2, 85748 Garching, Germany

${ }^{2}$ Departamento de Física, Universidad Carlos III de Madrid, 28911 Leganés, Madrid, Spain

${ }^{3}$ Laboratoire de Physique des Plasmas, Ecole Polytechnique, France

\begin{abstract}
:
In ASDEX Upgrade (AUG) the normalised gyroradius $\rho_{\star}$ was varied via a hydrogen isotope scan while keeping other dimensionless parameters constant. This was done in L-mode to minimise the impact of pedestal stability on confinement.

Power balance and perturbative transport analyses reveal that the electron heat transport is unaffected by the different isotope masses. Nonlinear simulations with the GENE code suggest that these L-mode discharges are ITG dominated. The different gyroradii due to the isotope mass do not necessarily result in a change of the predicted heat fluxes. This result is used in simulations with the ASTRA transport code to match the experimental profiles. In these simulations the experimental profiles and confinement times are reproduced with the same transport coefficients for hydrogen and deuterium plasmas. The mass only enters in the energy exchange term between electrons and ions.
\end{abstract}

These numerical observations are supported by additional experiments which show a lower ion energy confinement compared to that of the electrons. Additionally, hydrogen and deuterium plasmas have a similar confinement when the energy exchange time between electrons and ions is matched.

This strongly suggests that the observed isotope dependence in L-mode is not dominated by a gyroradius effect, but a consequence of the mass dependence in the collisional energy exchange between electrons and ions.

\section{Introduction}

Heat transport is a key issue in understanding fusion plasmas. It is well known that the transport processes in the main plasma cannot be described by collisional transport because the transport measured in experiments is anomalously high [1]. The transport which cannot be explained by neoclassical theory is attributed to turbulent effects, where the main turbulent mechanism can differ depending on the plasma regime. To understand and predict transport, parameter dependencies of the heat transport have been investigated over the years [2]. The most prominent experimental transport scalings are Bohm and gyro-Bohm. The difference between both scalings

* See the author list of "Overview of progress in European Medium Sized Tokamaks towards an integrated plasma-edge/wall solution" by H. Meyer et al., to be published in Nuclear Fusion Special issue: Overview and Summary Reports from the 26th Fusion Energy Conference (Kyoto, Japan, 17-22 October 2016) 
is $\chi_{\mathrm{gB}}=\rho_{\star} \chi_{\mathrm{B}}$ with the Bohm diffusivity $\chi_{\mathrm{B}}=T /(e B)$ and the normalized gyroradius $\rho_{\star}=\rho / a$ with $a$ as the plasma minor radius and $\rho=\sqrt{m T} / B$ as the gyro-radius. It should be noted that the mass dependence enters only with the gyro-radius in $\chi_{\mathrm{gB}}$ while $\chi_{\mathrm{B}}$ is independent of the mass. In mono-isotope studies H-modes are found to follow gyro-Bohm in the electron and ion channel while in L-mode the electrons are gyro-Bohm- and the ions Bohm-like [2] and references therein. The main difference between L- and $\mathrm{H}$-mode is the edge pedestal with its edge localised modes (ELMs). It might be possible that these differences have an impact on the transport scalings.

The original argument for the Bohm and gyro-Bohm scaling was motivated with the impracticality to scale dimensionally identical plasmas [3]. Dimensional identity would theoretically allow to predict confinement properties. To enable scalings to larger machines the size parameter $\rho_{\star}$ is allowed to vary. This is called dimensionally similar when the other dimensionless parameters are kept constant. The list of dimensionless parameters is long. Along with $\rho_{\star}$ the list includes normalised collisionality $\nu_{\star}$, normalized pressure $\beta$, the gradient lengths $R / L_{T}$ and $R / L_{n}$ with $L_{T}^{-1}=\nabla(\ln T)$, the aspect ratio, safety factor and more. For dimensional similar parameter scans exact knowledge of the scaling with $\rho_{\star}$ is most important. This is because the values for other dimensionless parameters - in particular, $\nu_{\star}$ and $\beta$ - needed for an ignited plasma have been achieved in present day experiments whereas $\rho_{\star}$ has to be extrapolated the most from the range already explored.

Gyro-Bohm theory assumes a scaling of the radial transport with a typical step length $\sim \rho$. So one consequence of a gyro-Bohm scaling is to expect the mass dependence of the gyro-radius to be found in the heat transport. This means plasmas with larger main ion mass should exhibit a higher heat transport. However, despite the expectations the mass dependence of heat transport is often found to go in the opposite direction [4]. This deviation between theory and observation is commonly referred to as the isotope effect of transport.

For predictions towards future fusion devices it is particularly important to solve the question of the isotope effect. Additional to the machine size, the ion mass in the plasma is a parameter that will change from today's experiments. While today mainly pure deuterium plasmas are studied, a fusion plasma will have a significant amount of tritium. To understand the impact of the mass of the hydrogen isotope on the plasma confinement multiple studies have been performed in the past. However, no satisfactory explanation for the observed mass dependence has been found. The experimental observations are summarized in the empirical scalings for the global thermal energy confinement time $\tau_{\mathrm{E}}$ as derived from the international confinement databases. The relevant dependencies are those for the heating power $P$ and the mass number $M$ which are similar for H-mode $\tau_{\mathrm{E}, 98(\mathrm{y} 2)} \propto P^{-0.69} M^{0.19}\left[5\right.$ ] and L-mode $\tau_{\mathrm{E}, \mathrm{L}} \propto P^{-0.73} M^{0.20}\left[6\right.$, where $\tau_{\mathrm{E}}$ is the thermal energy confinement time. The scaling laws show similar mass dependencies for L- and H-mode. This is not obvious because the ELM behaviour is observed to change significantly when changing the main ion mass [7,8] and it was shown recently that the amount of ELM energy losses can impact the pedestal and thereby the confinement [9]. Results from the JET DT campaign suggest that the mass dependence of edge/pedestal and core transport differ significantly, while the core transport is found to increase with mass the edge transport is reduced [10]. Also the boundary 


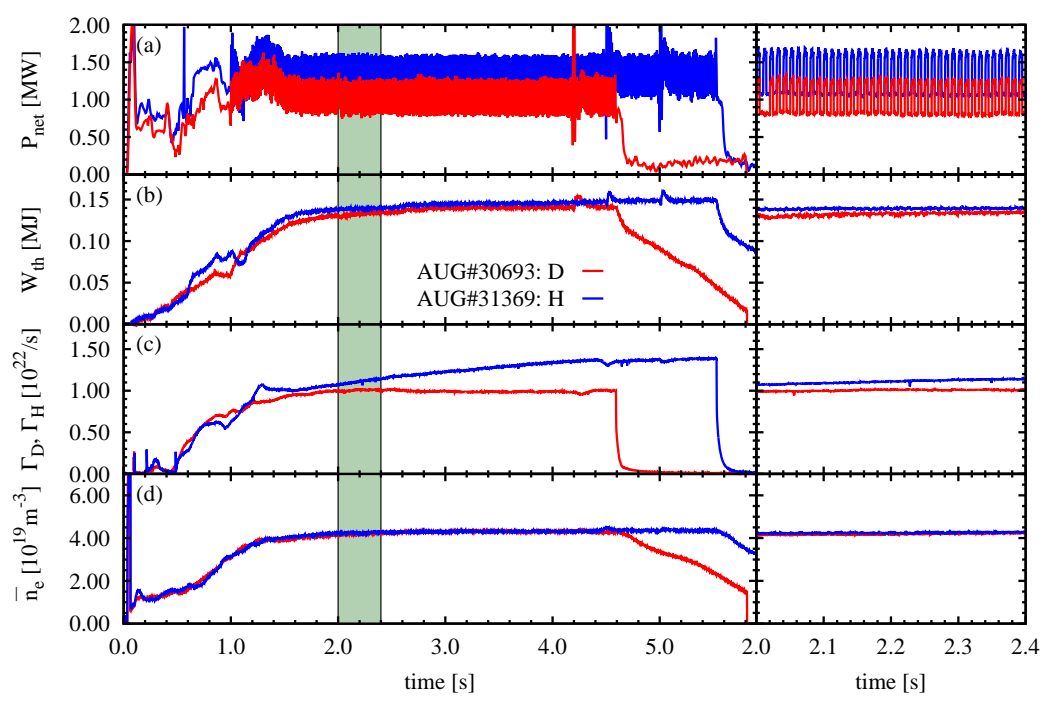

FIG. 1: Timetraces of heating power (a), thermal stored energy (b), gas puff level (c) and core line averaged density (d) for the discharge in deuterium (red) and in hydrogen (blue). On the right a zoom with the analysed time window is given.

between L- and H-mode the L-H transition depends on the isotope mass [11].

In order to separate ELM and edge stability from transport physics in the core this paper concentrates on L-mode plasmas performed in ASDEX Upgrade. The H-mode studies including the ELM physics are discussed in a different publication [12] where the main difficulties arising from the transition to $\mathrm{H}$-mode will be pointed out.

Even when restricting this study to L-mode plasmas, experimental difficulties arise from the multitude of effects a variation in the isotope mass can trigger. Changes in heat and particle confinement, edge stability and sawtooth behaviour are observed with different isotope masses. The causality between them is often not obvious. On top of that, secondary effects are introduced e.g. by the necessity of using different or additional heating systems which often do not have the same properties.

Considering this background it is not surprising that not all experiments concerning isotope scalings are found to be consistent. While most isotope studies show a reduced confinement or enhanced transport for hydrogen compared to deuterium [4, exceptions are found for L-modes. Beam heated L-modes in DIII-D show no effect of the main hydrogen isotope [7. Also in DIII-D L-modes the confinement reduction with lower isotope mass vanishes for $q_{95}<4.0$ [13]. In the ASDEX tokamak the magnitude of confinement difference between isotopes was found to vary with neutral beam power and vanished for the lowest power level [14]. The L-mode scaling for the thermal confinement time showed a $M^{0.20}$ mass dependence, however, when restricting the data base to limiter configurations no mass dependence is observed [6]. For stellarators no isotope dependence is found in the international database with variable heating schemes [15. However, when taking only electron heated L-mode discharges the ones with hydrogen show a lower confinement time than those with deuterium [16].

The theoretical understanding of the isotope effect is still developing. One crucial ingredient might be the zonal flow activity which is thought to regulate turbulence [17. Small scale residual zonal flows are supposed to exhibit an isotope dependence [18]. In particular, the geodesic acoustic 
mode (GAM), a variant of a zonal flow, was recently proposed as a possible player in explaining the isotope effect of transport. Probe measurements from smaller tokamaks suggest an isotope dependence of the GAM amplitude which would be consistent with the trends observed in transport [19-21]. For the discharges presented in this contribution similar measurements of the GAM activity were made with reflectometry, namely a lower GAM amplitude in the hydrogen plasma. The details of these measurements are discussed in a separate publication [22]. Another approach is provided by nonlinear gyrokinetic simulations with the GENE code which suggest the heat flux scales weaker with $\rho_{\star}$ than expected by gyro-Bohm [23]. Therefore, deviations from the gyroBohm scaling are not necessarily incompatible with gyro-kinetic theory. Also measurements of turbulence correlation lengths suggest that small scale turbulence ( $\left.5 \rho_{\mathrm{i}}\right)$ do scale with the main ion mass or $\rho_{\star}$, while mesoscale structures $\left(15-25 \rho_{\mathrm{i}}\right)$ do not [22]. Structures on this scale are also observed in numerical simulations with the gyrokinetic simulations [24, 25]. The relevance of these structures in the turbulence in terms of heat transport is a field of active research.

In section 2 we present dimensionally similar plasmas with different $\rho_{\star}$ which is achieved by varying the main ion mass and matching the kinetic profiles. The experimental approach (section 2.1) is presented with a detailed analysis of the heat transport section 2.2. To support the experimental observations nonlinear gyrokinetic simulations performed with the GENE code are discussed in section 2.3. To translate the Gene results into global plasma parameters Astra simulations are presented in section 2.4. In section 3 the impact of the heating mix on the confinement loss in hydrogen plasmas is quantified. In section 4 the discussion is extended to a broader data set of L-mode discharges. An overview and discussion of the results is given in section 5

\section{Isotope scan}

\section{$2.1 \quad$ Experiment}

The experimental strategy is to achieve a match in the kinetic profiles for two plasmas with different hydrogen isotopes as main ions. The profile match minimises effects on transport due to $\nu_{\star}, \beta$ and the gradient lengths $L_{T}^{-1}=-\nabla(\ln T)$ and $L_{n}^{-1}=-\nabla(\ln n)$, thereby, allows to identify any influence the mass number has on transport and confinement.

The density in these discharges is chosen to be relatively high to allow for turbulence measurements with reflectometry, additionally, for this density range discharges are very reproducible in AUG. One important consequence of the higher density is that the collisionality is increased, resulting in an improved coupling between electron and ion heat transport channels. At a given density the heating power and thereby the temperature is limited by the prerequisite to stay in L-mode. The discharges that achieved the best profile match were run with a plasma current of $I_{\mathrm{p}}=0.80 \mathrm{MA}$ and a toroidal field of $B_{\mathrm{t}}=-2.46 \mathrm{~T}$ resulting in a safety factor of $q_{95}=5.07$.

The L-mode discharges described in this section are AUG\#30693 with deuterium and AUG\#31369 hydrogen as main gas. The isotope purity is above $90 \%$ for both cases as measured with neutral particle analysis. They were very reproducible as they are exemplary for a series of several discharges each in deuterium and hydrogen. An overview of important time traces is shown in figure 

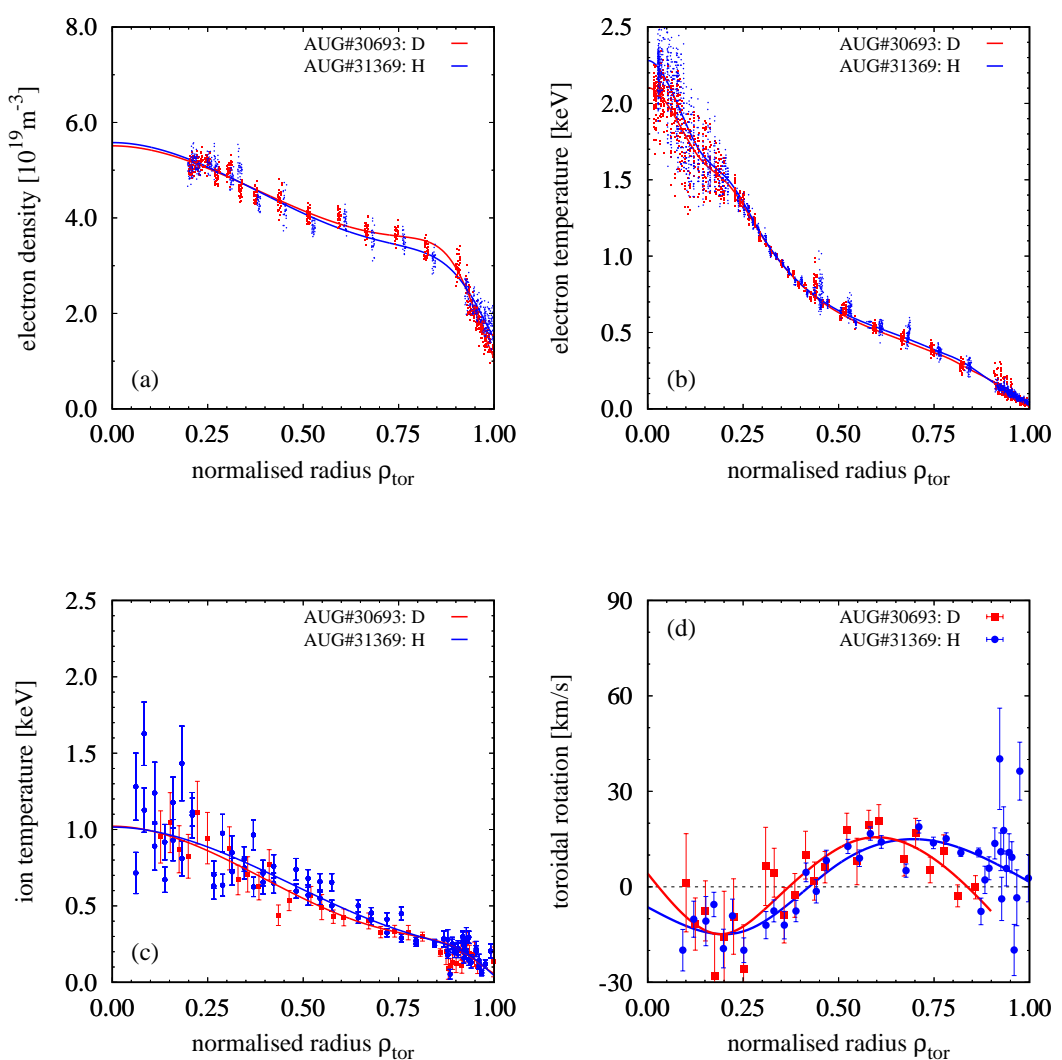

FIG. 2: Radial profiles of electron density (a), electron temperature (b), ion temperature (c) and toroidal rotation (d) for the discharge in deuterium (red) and in hydrogen (blue). The lines are fits to the data.

1. The power modulation of $P_{\text {net }}$ shown in figure 1 (a) is applied for perturbative transport analysis which will be described in section 2.2.3. Here $P_{\text {net }}=P_{\mathrm{OH}}+P_{\mathrm{aux}}-\mathrm{d} W / \mathrm{d} t$ with the ohmic power $P_{\mathrm{OH}}$, auxiliary heating $P_{\text {aux }}$ by electron cyclotron resonance heating $(\mathrm{ECRH})$ [26] or neutral beam injection (NBI) and the stored energy $W$. A near perfect profile match was achieved with only ECRH as auxiliary heating source. The profiles for electron density $n_{\mathrm{e}}$, electron temperature $T_{\mathrm{e}}$, ion temperature $T_{\mathrm{i}}$ and toroidal rotation $v_{\text {tor }}$ are shown in figure 2 The density is obtained with the Thomson scattering diagnostic (TS) [27] for the core and the Li-beam diagnostic [28] for the edge. The electron temperature is measured with electron cyclotron emission spectroscopy (ECE) 29] and TS, the data is synchronised to sawteeth and in figure 2 (b) only pre-crash data is shown. The ion temperature (c) and toroidal rotation (d) are measured with the charge exchange recombination spectroscopy (CXRS) [30,31]. The reversal of the toroidal rotation in the center is a typical observation in AUG for the given collisionality regime and plasmas without external momentum input [32]. The match of $v_{\text {tor }}$ suggests the intrinsic rotation is unaffected by the isotope mass. The uncertainties of the ion temperature and toroidal rotation measurement are relatively large because of the low CXRS signal. The reason for the low signal is twofold, first only short neutral beam blips could be used to avoid entering H-mode, second the L-mode plasmas had a quite low impurity content. From the center up to mid-radius the effective charge $Z_{\text {eff }}$ is close to 1 as determined from CXRS and bremsstrahlung [33]. Both the hydrogen and deuterium discharge have comparable impurity contents up to $\rho_{\text {tor }} \sim 0.7$, where $\rho_{\text {tor }}$ is the square root of the normalized toroidal flux, for the edge $\left(\rho_{\text {tor }}>0.7\right)$ the discharge in hydrogen shows a lower 
impurity level. This is consistent with measurements by the bolometers [34], in the deuterium discharge a radiating mantle is forming in the edge region which is not present in the hydrogen discharge. However, the total radiated power is on a very low level, $150 \mathrm{~kW}$ in deuterium and 100 $\mathrm{kW}$ in hydrogen, the difference is less than $5 \%$ of $P_{\text {net }}$.

The equilibrium used for the mapping and the modeling is calculated with an advanced analysis method including the thermal pressure profile and the sawtooth inversion radius as additional constraints [35]. This improves the estimate for the magnetic shear which can be crucial for gyrokinetic simulations. The lines shown in figure 2 for $T_{\mathrm{e}}, T_{\mathrm{i}}, v_{\mathrm{tor}}$ are spline fits, for $n_{\mathrm{e}}$ it is a modified tanh fit (edge) and a 2 nd order polynomial in the core. The fits of $T_{\mathrm{e}}, T_{\mathrm{i}}, n_{\mathrm{e}}$ use the boundary condition of $\partial f / \partial \rho_{\text {tor }}=0$ at $\rho_{\text {tor }}=0$, where $f=T_{\mathrm{e}}, T_{\mathrm{i}}, n_{\mathrm{e}}$. These fits will be used to calculate all derived quantities.

The mean ECRH power is $0.50 \mathrm{MW}$ for deuterium and $0.81 \mathrm{MW}$ for hydrogen, it consisted in both cases of a constant source deposited on axis and a modulated source off axis. The latter was applied for the heat pulse transport study which is discussed in section 2.2.3. Adding the ohmic heating and taking the different core radiation levels into account the power through the separatrix is $P_{\text {sep }}^{\mathrm{D}}=1.06 \mathrm{MW}$ and $P_{\text {sep }}^{\mathrm{H}}=1.39 \mathrm{MW}$. This means that in deuterium $76 \%$ of the heating power in hydrogen is needed to achieve the same stored energy in the plasma. The L-mode scaling law is $\tau_{\mathrm{E}, \mathrm{L}} \propto P^{-0.73} M^{0.20}$ [6] and so for the stored energy $W \propto P^{0.27} M^{0.20}$ is expected. Now if the stored energy should be matched for different isotopes $W_{\mathrm{D}}=W_{\mathrm{H}}$ the fairly weak mass contribution is enhanced by the additional power degradation $P \propto M^{-0.20 / 0.27}=M^{-0.74} \Rightarrow(2 / 1)^{-0.74}=0.60$. The measured and expected heating ratio follow the same trend, in fact the discharge with hydrogen main ions performs better than expected from the scaling. In terms of the confinement scaling factors one gets: $L^{\mathrm{D}}=\tau_{\mathrm{E}}^{\mathrm{D}} / \tau_{\mathrm{E}, \mathrm{L}}=1.02, L^{\mathrm{H}}=1.16$ - as comparison the more familiar H-mode scaling: $H_{98(\mathrm{y} 2)}^{\mathrm{D}}=\tau_{\mathrm{E}}^{\mathrm{D}} / \tau_{\mathrm{E}, 98(\mathrm{y} 2)}=0.67, H_{98(\mathrm{y} 2)}^{\mathrm{H}}=0.77$.

The parameter that could not be matched perfectly in the mass scan was the sawtooth behaviour. The details are discussed in section 2.2 .2 and the differences are taken into account for the interpretative modeling.

Another important parameter is the amount of gas fuelling necessary to match the density. In contrast to H-mode, where an order of magnitude larger gas puffing is required in hydrogen to achieve similar densities as in deuterium [12], the difference in gas puff is quite small in L-mode. Only $0-30 \%$ larger gas puff is required which is within variations to be expected depending on wall conditions. So there is no direct evidence of an isotope dependence of particle confinement in L-mode.

The discharges described above meet the requirement of minimal variation in $\nu_{\star}$ and $\beta$ and the normalized gradient lengths $R / L_{T}$ and $R / L_{n}$. The corresponding radial profiles are illustrated with their uncertainties in figure 3 and figure 4 . The gradient lengths and their uncertainties are determined on radial windows of $3 \mathrm{~cm}$ length. 

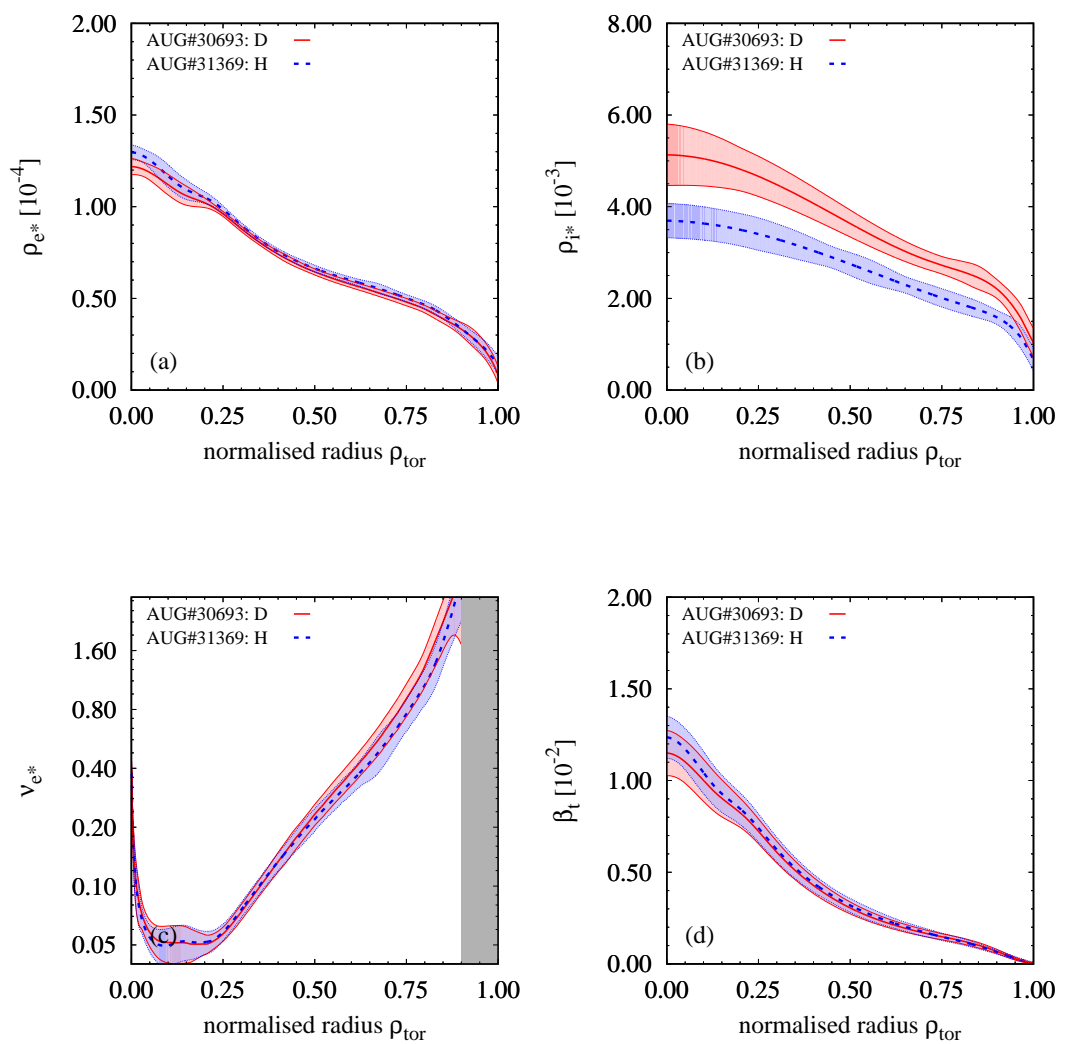

FIG. 3: Radial profiles of the normalized gyroradius for electrons (a) and ions (b), the collisionality on logarithmic scale (c) and the normalized total pressure (d).
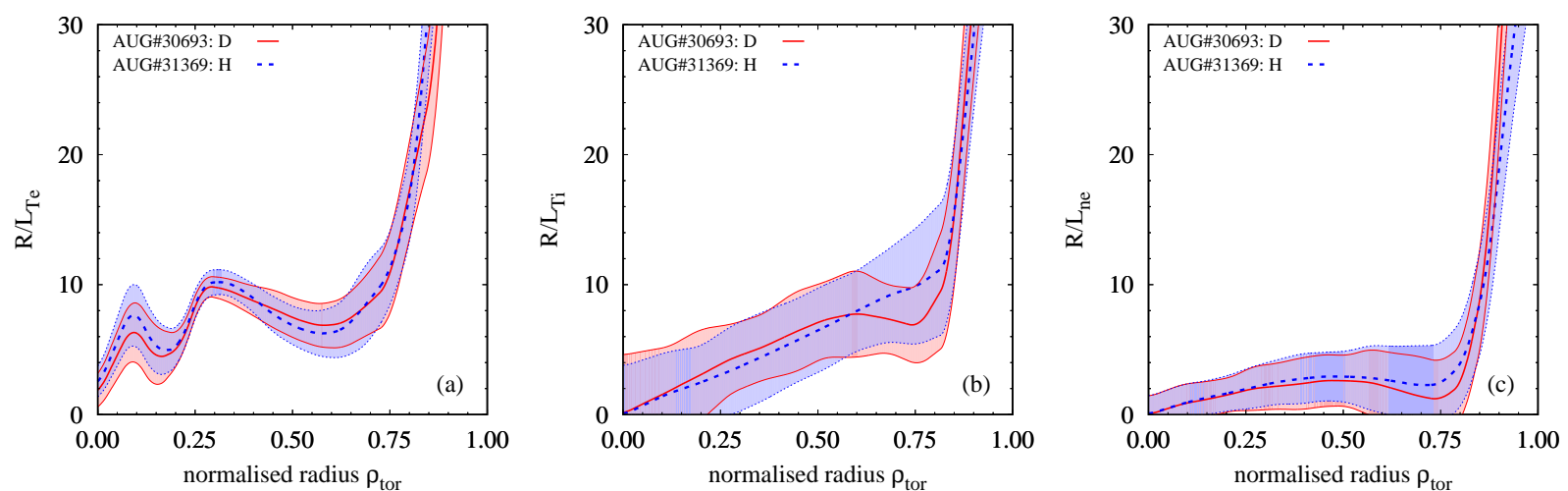

FIG. 4: Normalized gradient lengths of electron temperature (a), ion temperature (b) and electron density (c) based on the profiles shown in figure 2 and determined on radial windows of $3 \mathrm{~cm}$ length. 

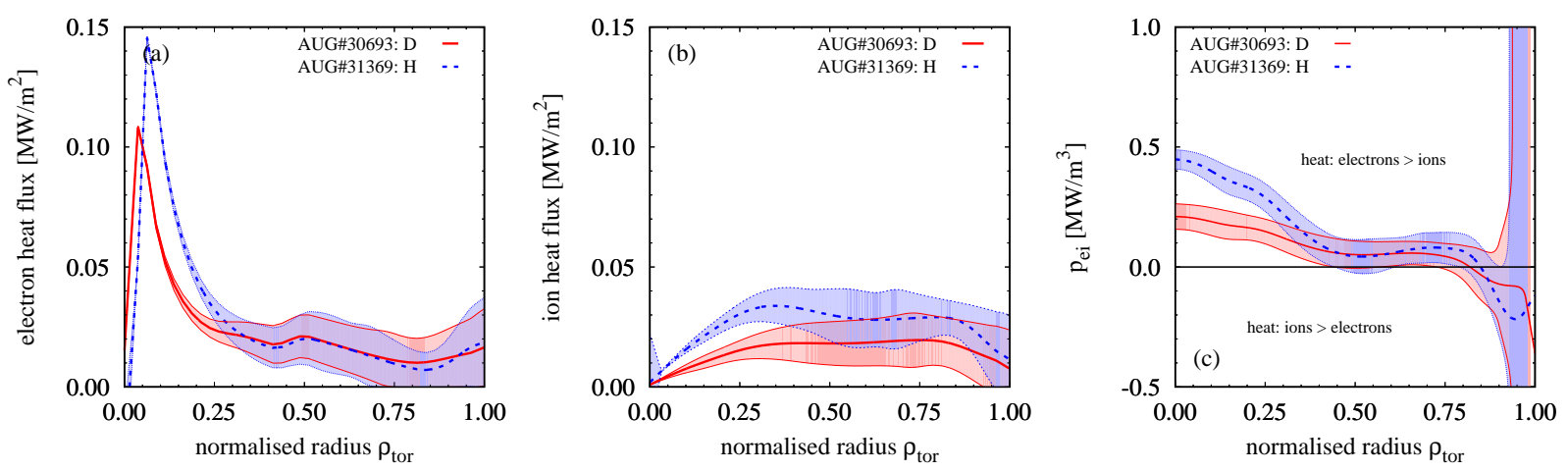

FIG. 5: Radial profiles for heat fluxes of electrons (a) and ions (b). The electron-ion heat exchange term is plotted in (c).

\subsection{Transport analysis}

\subsubsection{Power Balance}

The main tool for the power balance (PB) analysis is the TRAnsP code [36]. It is used to determine the heat flux profiles for electrons and ions. The input profiles are those discussed in section 2.1 The radiation and $Z_{\text {eff }}$ profiles are taken into account. The impact of the sawteeth on the heat flux in TRAnsP is treated based on the Kadomtsev model and assumes complete magnetic reconnection inside the $q=1$ surface [37. The resulting electron $q_{\mathrm{e}}$ and ion heat fluxes $q_{\mathrm{i}}$ are shown in figure 5 (a) and (b). Despite the different ECRH power levels in both discharges the electron heat fluxes match well for $\rho_{\text {tor }}>0.3$. The reason for this is the mass dependence of the electron ion heat transfer term [38] illustrated in figure 5 (c)

$$
p_{\mathrm{ei}}=\frac{3 m_{\mathrm{e}}}{m_{\mathrm{i}}} \frac{n}{\tau_{\mathrm{e}}}\left(T_{\mathrm{e}}-T_{\mathrm{i}}\right) \propto \frac{Z_{\mathrm{i}}^{2}}{m_{\mathrm{i}}} \frac{n^{2}}{T_{\mathrm{e}}^{3 / 2}}\left(T_{\mathrm{e}}-T_{\mathrm{i}}\right)
$$

where $\tau_{\mathrm{e}}$ is the collision time for the electrons and $Z_{\mathrm{i}}$ the ion charge number. Although only electron heating is applied the heat fluxes are regulated by the electron-ion energy exchange which is two times stronger in hydrogen compared to deuterium. Therefore, the additional electron heating in hydrogen is in fact transported by the ion channel. This results in different $q_{\mathrm{i}}$ for the two isotopes as shown in figure 5 (b) with $q_{\mathrm{i}}^{\mathrm{H}}>q_{\mathrm{i}}^{\mathrm{D}}$ and $q_{\mathrm{i}}^{\mathrm{H}}>1.5 q_{\mathrm{e}}^{\mathrm{H}}$ for most of the radius. The uncertainties in the heat fluxes originate from the uncertainties in the heat transfer term which accumulate over the radius. Therefore, it should be noted that the uncertainties of $q_{\mathrm{e}}$ and $q_{\mathrm{i}}$ are not independent of each other, in particular, the sum $q_{\mathrm{e}}+q_{\mathrm{i}}$ is known very accurately.

The match $q_{\mathrm{e}}^{\mathrm{H}}=q_{\mathrm{e}}^{\mathrm{D}}$ is not a coincidence, but a consequence of the goal to match the electron temperatures. The difference in heating power for the $T_{\mathrm{e}}$ match is due to the differences in $p_{\mathrm{ei}}$. So the observations made here might be valid only for a certain collisionality and $T_{\mathrm{e}} / T_{\mathrm{i}}$ domain. In particular, the strong density dependence in $p_{\mathrm{ei}}$ is important. This is because the heat transfer term looses importance for lower densities which will be discussed in section 4 . The density in the mass scan is high enough such that $p_{\mathrm{ei}}$ has a significant impact on $q_{\mathrm{e}}$ and $q_{\mathrm{i}}$. The consequence of the higher heating power and larger $p_{\mathrm{ei}}$ in hydrogen is $q_{\mathrm{i}}^{\mathrm{H}}>q_{\mathrm{i}}^{\mathrm{D}}$. In this experiment the ion heat flux was not an external control parameter, the experiment with $q_{\mathrm{i}}$ control is discussed in section 

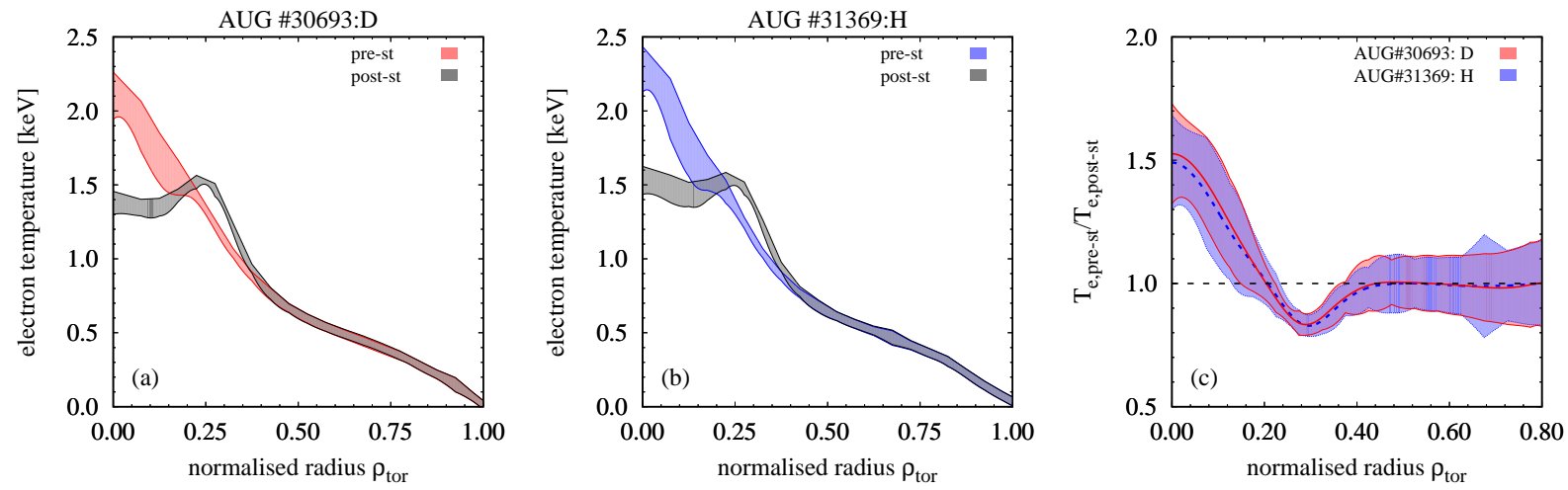

FIG. 6: Radial profiles of the electron temperature before and after a sawtooth crash for deuterium (a) and hydrogen (b). The magnitude of the crash is shown in (c) as the ratio of temperatures before and after the crash.

\section{3.}

There are three direct results of the power balance analysis. First, $q_{\mathrm{i}}$ shows no gyro-Bohm dependence because for the gyro-Bohm scaling $\sqrt{2} q_{\mathrm{i}}^{\mathrm{H}}=q_{\mathrm{i}}^{\mathrm{D}}$ is expected. Second, the electron heat transport and therefore, the electron temperature is not affected by the changes in the ions. Third, $\rho_{\star}$ or the isotope mass is not the only parameter that changed in the experiment, the ion heat flux changed as well with $q_{\mathrm{i}}^{\mathrm{H}} / q_{\mathrm{i}}^{\mathrm{D}}=1.5$ at $\rho_{\text {tor }}=0.6$. The resulting power balance diffusivities are varying over the radius. For the electrons they are $\chi_{\mathbf{e}}^{\mathrm{PB}}=0.5-1.0 \mathrm{~m}^{2} / \mathrm{s}$ where the $\chi_{\mathbf{e}}^{\mathrm{PB}}$ for hydrogen equals the one of deuterium within this range. The uncertainties in the ion temperature gradients are too large to distinguish between hydrogen and deuterium, both gases show a $\chi_{\mathrm{i}}^{\mathrm{PB}}=1-3 \mathrm{~m}^{2} / \mathrm{s}$.

\subsubsection{Sawteeth}

As indicated in the experimental overview the sawtoothing behaviour changes in the isotope scan. The sawtooth frequencies differ between both discharges $f_{\mathrm{st}}^{\mathrm{D}}=85 \mathrm{~Hz}$ and $f_{\mathrm{st}}^{\mathrm{H}}=120 \mathrm{~Hz}$. This change in sawtooth frequency is typically observed in isotope studies with a similar dependence of $f_{\text {st }} \sim A^{-0.5}$ [4]. The impact on the ion temperature was not measured, but is supposed to be small because the ion temperature is comparably flat within the sawtooth inversion radius. In this section it will be shown that all observed differences are a direct consequence of the higher heating power power in hydrogen.

Figure [6 shows sawtooth synchronised electron temperature profiles before and after the crash for deuterium (a) and hydrogen (b). The pre- and post-crash temperature ratio figure 6 (c) nicely illustrates that the size of the sawteeth does not change with the isotope mass. Additionally, the sawtooth precursor mode has similar characteristics in amplitude and frequency $(\sim 2 \mathrm{kHz})$ for both discharges.

With the fitted kinetic profiles and the known sawtooth frequency the temporal evolution of the central stored energy $\Delta_{W}=\mathrm{d} W / \mathrm{d} t$ during the profile recovery is calculated. The temperature profiles in hydrogen recover faster than in deuterium by an amount of $\Delta_{W}^{\mathrm{H}}-\Delta_{W}^{\mathrm{D}}=(84 \pm 41) \mathrm{kJ} / \mathrm{s}=$ $(84 \pm 41) \mathrm{kW}$. The faster profile recovery is consistent with the effective electron heating within the $q=1$ surface which is $100 \mathrm{~kW}$ larger for hydrogen. The faster temperature evolution allows the 

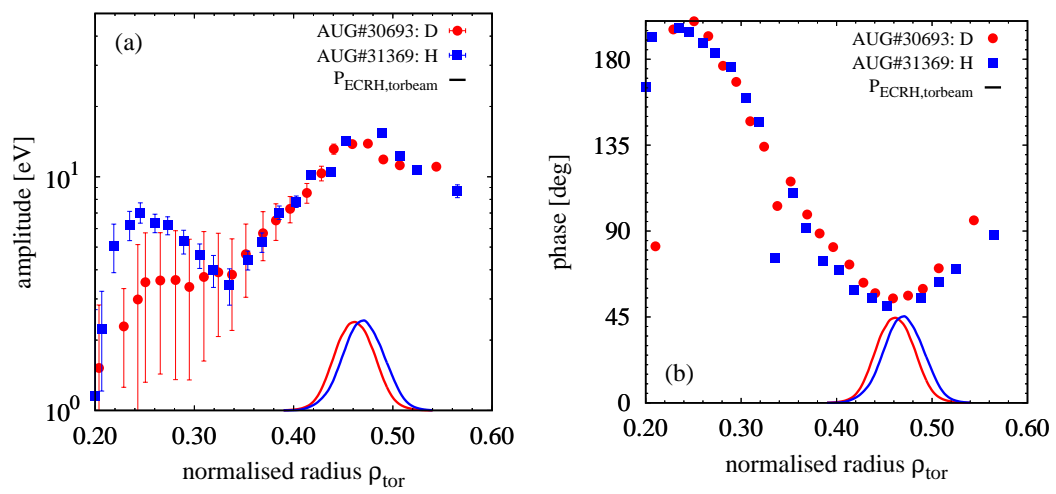

FIG. 7: Radial profiles for amplitude (a) and phase (b) of temperature perturbations imposed by 100 Hz ECRH modulation. The ECRH deposition profiles obtained from TORBEAM calculations are indicated with solid lines.

crash condition to be reached earlier which explains the shorter period in hydrogen. Therefore, no other explanation is necessary to understand the observed difference in sawtooth frequencies. Also no other influencing factor is expected, as the kinetic profiles are matched nearly perfectly and the $q=1$ surface is at same position. Therefore, relevant parameters like the shear or the resistivity are also the same.

\subsubsection{Perturbative transport analyses}

The heat pulse (HP) analysis using ECRH to determine transport properties is a well established method [39, 40]. The ECRH is used to induce small localized perturbations, their response in the electron temperature is measured over the radius. The perturbation is applied with a repetition rate of $\omega=100 \mathrm{~Hz}$ and the response is measured with the sampling rate of $32 \mathrm{kHz}$ of the ECE diagnostic. The position of the modulated ECRH is off-axis at $\rho_{\text {tor }} \sim 0.46$. In the deuterium plasma this gyrotron delivers on average $220 \mathrm{~kW}$ compared to $280 \mathrm{~kW}$ deposited on-axis, in hydrogen 290 $\mathrm{kW}$ are heated off-axis and $550 \mathrm{~kW}$ on-axis.

The Fourier analysis of the temperature perturbation yields radial amplitude $A$ and phase $\phi$ profiles. These are used to characterise the heat pulse diffusion $\chi^{\text {HP }}$ which is calculated for slab geometry with cylindrical corrections [41,42]

$$
\chi_{\mathrm{e}}^{\mathrm{HP}}=\frac{\frac{3}{4} \omega}{\nabla \phi\left(\frac{\nabla A}{A}+\frac{1}{2} r\right)}
$$

where $r$ the minor radius of the measurement location.

The comparison of amplitude and phase profiles for the $\rho_{\star}$ mass scan is shown in figure 7 The central region $\rho_{\text {tor }}<0.35$ of the plasma is influenced by sawtooth crashes. However, for the outer radii their impact on the perturbation amplitude is negligible which is observed in the individual spectra. The profiles of amplitude and phase around the ECRH deposition illustrate that there is no difference in the electron heat pulse propagation despite the different isotopes. The perturbation of the electron temperature is only $14 \mathrm{eV}$ or $2 \%$ at the heat deposition, so it is small and Eq. (2) can be applied. The derived diffusivities for $\rho_{\mathrm{tor}} \in[0.34,0.44]$ are $\chi_{\mathrm{e}, \mathrm{H}}^{\mathrm{HP}}=2.4 \pm 0.9 \mathrm{~m}^{2} / \mathrm{s}$ and $\chi_{\mathrm{e}, \mathrm{D}}^{\mathrm{HP}}=2.2 \pm 0.3 \mathrm{~m}^{2} / \mathrm{s}$. Since $\chi^{\mathrm{HP}}$ reflects $\partial q_{\mathrm{e}} / \partial\left(\nabla T_{\mathrm{e}}\right)$ these measurements extend the result that 
electron heat transport is not affected by the ion mass.

\subsection{Transport modeling with GENE}

The expected turbulence present in the plasma is determined via simulations with the GENE code [43]. GENE solves self-consistently the gyrokinetic-Maxwell system of equations on a fixed grid in a five dimensional phase space and time using a $\delta f$ scheme. Two velocity coordinates $\left(v_{\|}, \mu\right)$ and three spatial field-aligned coordinates $(x, y, z)$ are used. Here, $z$ is the coordinate along the magnetic field line, which contains the description of the magnetic geometry. The radial coordinate $x$ and the binormal coordinate $y$ expand a radially local volume in the neighbourhood of the reference magnetic surface, labeled with the effective radius $\rho_{\text {tor }}$. This is known as the flux tube approximation, and in axisymetric systems describes the full magnetic surface. The boundary conditions in $(x, y)$ allow for a Fourier representation denoted by $\left(k_{x}, k_{y}\right)$, which is computationally much more convenient. We perform three kinetic species simulations (ions, electrons and impurities) include electromagnetic effects, collisions and the macroscopic $\mathrm{E} \times \mathrm{B}$ shear flow present in AUG.

In linear simulations, we use $n_{x}=9$ points in the radial position, one mode in the $y$ direction and 32 points in $z$. The magnetic equilibrium geometry is taken from the TRACER-EFIT interface [44]. Since we consider collisions, the grid in velocity space requires $n_{v}=64$ and $n_{\mu}=16$ points, instead of the usual $n_{\mu}=8$ in collisionless simulations.

Nonlinear (turbulent) runs require $n_{k x}=128$ and $n_{k y}=48$ modes in Fourier space, while the other grid dimensions remain unchanged. In real space, the box size of $(x, y)$ is $\approx 125 \rho_{s}$ each, being $\rho_{\mathrm{s}}$ the sound gyroradius. In this way we resolve a range of $k_{y} \cdot \rho_{\mathrm{s}} \in(0,2.2)$, covering the ion temperature gradient (ITG) and trapped electron mode (TEM) scales. The electron temperature gradient (ETG) turbulence is found to be negligible for the global power balance in these scenarios. Since we are using the $\delta f$ and local approximations, the simulations are gradient driven, in the sense that the transport is created by the prescribed background gradients of density and temperature provided by the experimental data. Exhaustive convergence tests have been run to fix those numerical parameters. Each of the nonlinear runs presented in this work requires around 80.000 CPU hours to run. In this way we have enough time in the turbulence saturated phase to obtain meaningful statistical observations.

To illustrate the trends resulting from gyrokinetics an arbitrary scan of the ion temperature gradient length $L_{T i}$ was performed around the experimental data. The gradient scans in linear simulations suggest negligible influence of $L_{T \mathrm{e}}$ and $L_{n}$ on the outcome of the nonlinear simulation. The ITG drive is the dominant turbulent mechanism for these discharges.

The main result of the nonlinear $L_{T \mathrm{i}}$ scan is shown in figure 8 with the experimental measurements for two different radial positions in the plasma. In figure 8 the total heat fluxes $Q_{\text {tot }}=Q_{\mathrm{e}}+Q_{\mathrm{i}}$ are plotted against the inverse ion temperature gradient length $R / L_{T \mathrm{i}}$, here $Q=q A$. As expected these GENE simulations show that ITG turbulence reacts very sensitively to changes in $L_{T \mathrm{i}}$. In figure 8 the heat fluxes in deuterium expected by the gyro-Bohm scaling, for a given hydrogen heat flux, are indicated by the dashed lines. It is observed that the heat fluxes do not follow gyro-Bohm 

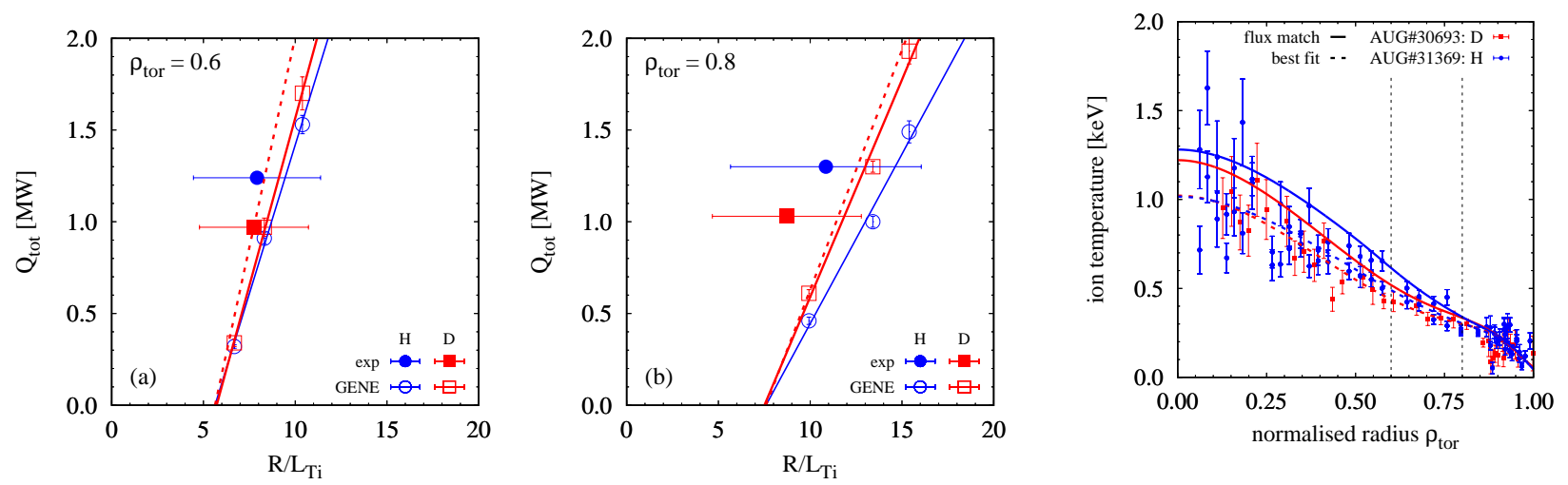

FIG 8: Total heat fluses plotted against the inverse ion temperature gradient length for two different radii. Open symbols are nonlinear GENE simulations and full symbols data (dashed lines) and two possible proexperimental measurements. The solid lines are fits to the simulated fluxes and the files with the $L_{T \mathrm{i}}$ of the GENE flux match dashed line represents $\sqrt{2} Q^{\mathrm{H}}$.

at $\rho_{\text {tor }}=0.6,0.8$ (solid lines).
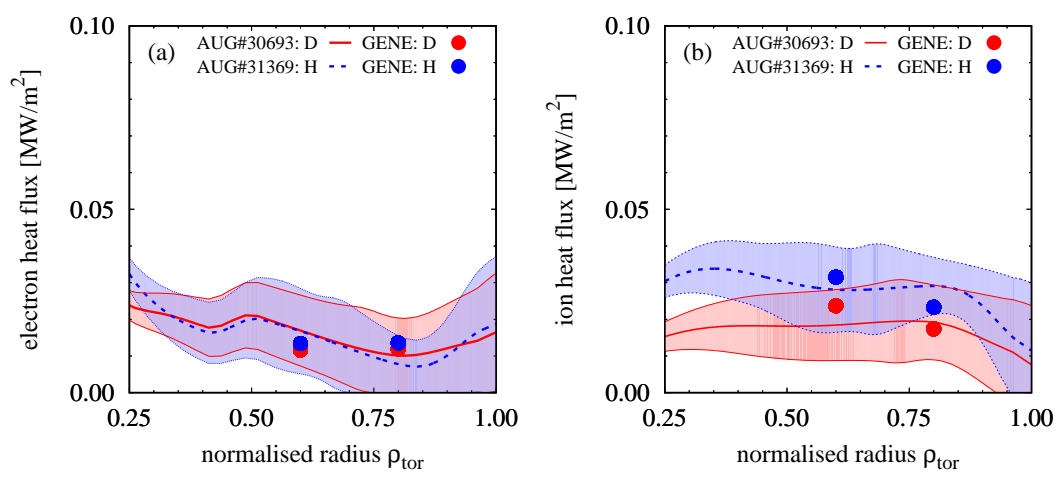

FIG. 10: Individual heat flux channels of electrons (a) and ions (b) with the experimental data from figure 5 as lines and the GENE values from the total flux match as points.

theory at $\rho_{\text {tor }}=0.6$ because $Q^{\mathrm{D}}<\sqrt{2} Q^{\mathrm{H}}$ while at the outer radii $\rho_{\text {tor }}=0.8$ the deviation from gyro-Bohm is smaller. For both radii the simulated fluxes do not follow the experimental trend $Q^{\mathrm{H}}>Q^{\mathrm{D}}$.

To illustrate the uncertainties, $T_{\mathrm{i}}$ profiles are reconstructed with a $L_{T \mathrm{i}}$ for which the simulation yields the experimental values of the total flux. The $L_{T i}$ profile is a spline fit using the two known values at radii $\rho_{\text {tor }}=0.6$ and 0.8 and additional boundary conditions using the experimental values of $T_{\mathrm{i}}\left(\rho_{\mathrm{tor}}=1.0\right), L_{T \mathrm{i}}\left(\rho_{\mathrm{tor}}=0.0\right)$ and $L_{T \mathrm{i}}\left(\rho_{\mathrm{tor}}=1.0\right)$. These boundary conditions are reasonable for L-mode plasmas. The results are shown in figure 9 and the $L_{T i}$ variation predicted by GenE to account for a $30 \%$ variation in heat fluxes is just within the experimental uncertainties. An independent measurement to test the differences in $T_{\mathrm{i}}$ is the stored energy, however, the impact on the stored energy is below $10 \%$ and also within the uncertainties. The relative difference between deuterium and hydrogen as expected by the simulation is well within the uncertainties of the CXRS measurements.

Above only total heat fluxes were considered because the experimental value of $Q_{\text {tot }}$ is most robust. However, the ITG turbulence does not only drive ion but also electron heat flux and in section 2.2.1 the importance of the individual transport channels was pointed out. If the total heat fluxes are matched with the experimental ones, GENE predicts a distribution for the heat fluxes 
which is dominated by the ion channel. For $\rho_{\text {tor }}=0.6$ the total heat flux match is well within the experimental uncertainties, for $\rho_{\text {tor }}=0.8$ the flux match is only achieved when pushing the local gradients to the utmost boundaries of the uncertainty. The importance of the ion channel is observed in hydrogen as well as in deuterium. In the simulation about $80 \%$ of the additional heat flux in the hydrogen discharge is going through the ion channel. The same trend is observed in the experiment and also the absolute values are consistent with the measurements within the uncertainties as shown in figure 10.

It has to be noted that the separation of heat fluxes in the ions for hydrogen and deuterium is no direct consequence of the different isotopes in the gyrokinetic simulations. It is a consequence of the variation in ion temperature gradient length to achieve the total flux match. This means if the total heat flux in the plasma increases and the transport channels are coupled the majority of the heat will be transported over the ion channel in an ITG dominated case. This prediction by GENE is also observed in experiment.

One criticism of these kind of gradient scans with the GENE code is that often the uncertainties of the measurement in $R / L_{\mathrm{T}}$ are enough to be consistent with a broad range of heat fluxes. Therefore, the data obtained here is used to show that the strong relation between temperature gradients and heat fluxes is compatible with experimental observations. To do so we estimate the power degradation in the ions expected due to ITG turbulence as predicted by GENE . The ion stored energy is determined from $T_{\mathrm{i}}$ profiles which are reconstructed from $R / L_{T \mathrm{i}}$ following the method described for figure 9 and neglecting possible edge contributions which is justified under L-mode conditions. As ion heating we take the ion heat flux from the simulation for a given $R / L_{T \mathrm{i}}$. This directly gives the power degradation of the ion energy confinement time $\tau_{\mathrm{E}, \mathrm{i}} \propto P_{\mathrm{i}}^{-0.64 \pm 0.10}$ which is similar to the power degradation in the global confinement time scaling. To test the quality of this result the exponent $\alpha_{P}$ for the power degradation is determined for different values of transport stiffness. Assuming a stiffness half the GENE value gives $\alpha_{P}=-0.44$ and with twice the value $\alpha_{P}=-0.76$. Therefore, the profile stiffness deduced from the GENE results seems to be at the lower end of the spectrum which is still compatible with the experimental scaling law. Weaker stiffness deviates significantly from the experimental values while stronger stiffness remains within the experimental uncertainties.

The power degradation of the global confinement time $\tau_{\mathrm{E}}$ cannot be inferred directly because Gene does not predict the $T_{\mathrm{e}}$ profile response on additional electron heat flux as this would require multi-scale simulations in order to assess the fine-scale (ETG) turbulence contribution.

\subsection{Transport modeling with AsTRA}

In the previous section gyrokinetic simulations were discussed which showed that a change in isotope does not necessarily result in a change of heat transport. In this section we will describe predictive AsTRA [45] simulations which show that a reduction of confinement is possible with the same transport coefficients.

For this purpose the critical gradient transport model is used as heat diffusivity for both electrons 

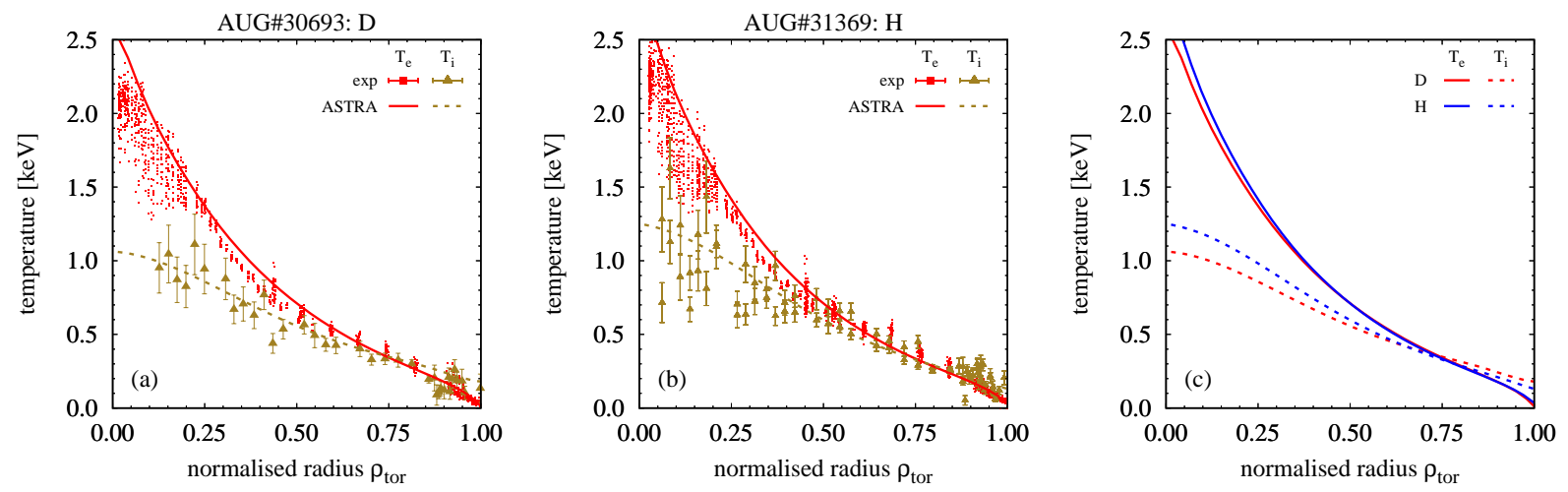

FIG. 11: Experimental data (points) and simulated temperature profiles (lines) using the critical gradient model in the ASTRA transport code. The deuterium plasma is shown in (a), the hydrogen plasma in (b) and a comparison of the simulated profiles in (c).

and ions [6]

$$
\chi=\chi_{\mathrm{s}} q^{3 / 2} \frac{T}{e B} \frac{\rho_{\mathrm{s}}}{R}\left(\frac{R}{L_{T}}-\kappa_{\mathrm{c}}\right) H\left(\frac{R}{L_{T}}-\kappa_{\mathrm{c}}\right)+\chi_{0} q^{3 / 2} \frac{T}{e B} \frac{\rho_{\mathrm{s}}}{R}
$$

with the stiffness parameter $\chi_{\mathrm{s}}$, the safety factor $q$, the critical gradient $\kappa_{\mathrm{c}}, H(x)$ as a Heaviside function and $\chi_{0}$ the parameter for the transport below the threshold. There is an explicit mass dependence in the gyroradius $\rho_{\mathrm{s}}$, but, both simulations use the same mass for the gyroradius, the one of deuterium. The transport coefficients are the same for both gases. For the electrons constant values are chosen $\left(\chi_{\mathrm{s}}, \kappa_{\mathrm{c}}, \chi_{0}\right)=(0.3,4.0,0.1)$. These values are typical for AUG plasmas [47] and they are in good agreement with the measurements of the perturbative transport as described in section 2.2.3. The ion heat transport is set to be more stiff $\chi_{\mathrm{s}}=1.0 \mathrm{~m}^{2} / \mathrm{s}$ and with neoclassical values for $\chi_{0}$. For the critical gradient the values from the GENE simulations are taken along with a radial dependence as in the Weiland model [48]. The main contribution to the radial dependence is via $T_{\mathrm{e}} / T_{\mathrm{i}}$ and reduces $\kappa_{\mathrm{c}}$ for a larger ratio which is consistent with results from AUG [49].

With the experimental heat fluxes the model reproduces the experimental profiles nearly perfectly. For deuterium this is illustrated in figure11(a) and for hydrogen in figure11(b). A comparison of the simulated profiles in hydrogen and deuterium is shown in figure 11(c). The differences between both simulations are the higher ECRH power for the hydrogen plasma and the energy exchange term between electrons and ions Eq. (1), which is twice as efficient in hydrogen compared to deuterium. The match of simulated and measured profiles using the experimental heating powers directly means that also the global confinement parameters are reproduced by the model. In particular, we find a reduction of the confinement time in the hydrogen plasma with $\tau_{\mathrm{E}}^{\mathrm{H}}=0.75 \tau_{\mathrm{E}}^{\mathrm{D}}$. The higher $\chi_{\mathrm{i}}$ in the case of hydrogen is a consequence of the higher heat flux in the ion channel and not a mass dependence of the heat transport.

Although, the $T_{\mathrm{i}}$ measurements are not accurate enough to completely exclude a dependence between heat transport and isotope mass. It is sufficient to have the same transport coefficients for hydrogen and deuterium to reproduce the experimental profiles and global confinement properties. Only the well known mass dependence of the collisional energy exchange between electrons and ions has to be taken into account. 


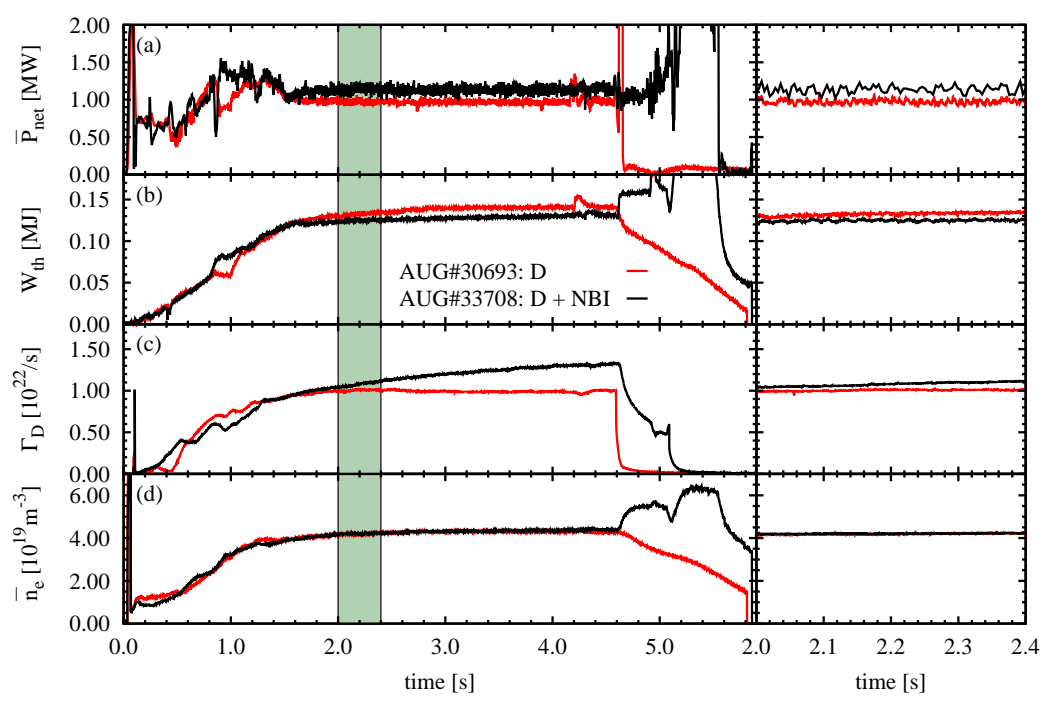

FIG. 12: Timetraces of time averaged heating power (a), thermal stored energy (b), gas puff level (c) and core line averaged density (d) for the electron heated case (red) and the one with direct ion heating (black). On the right a zoom with the analysed time window is given.

\section{Change of electron to ion heating ratio}

As discussed in the previous sections a change of the isotope mass will also vary the distribution of electron vs. ion heat flux even in a purely electron heated case. The ratio of electron and ion heat flux can also be varied by means of auxiliary heating in a single plasma with just one main ion species.

In figure 12 the time traces of the ECRH only discharges discussed in section 2.1 are shown again with an additional discharge in black with partly direct ion heating by NBI. The aim was to achieve an identity match in the same gas with different heating methods. The new discharge AUG $\# 33708$ used $P_{\mathrm{NBI}}=0.56 \mathrm{MW}$ and $P_{\mathrm{ECRH}}=0.16 \mathrm{MW}$. The first observation is that $P_{\mathrm{sep}}^{\mathrm{D}, \mathrm{NBI}}=1.24 \mathrm{MW}$ is necessary for a profile match which corresponds to $P_{\mathrm{sep}}^{\mathrm{D}, \mathrm{NBI}} / P_{\mathrm{sep}}^{\mathrm{D}, \mathrm{ECRH}}=1.18$ meaning more power is applied with NBI. The corresponding profiles are shown in figure 13 In particular, the ion temperature is matched extremely well despite $0.3 \mathrm{MW}$ direct ion heating due to the NBI. Although, the line averaged density matches perfectly, the density profile with direct fuelling by the NBI and reduced ECRH power is less peaked [50]. The core toroidal rotation is positive due to the torque induced by the NBI but towards the edge the rotation is on a similar level for all discharges. Overall, the dimensionless match is quite good. Yet, significant differences in the confinement are observed.

Since the stored energy is the same for different heating levels this would result in a power degradation of the global confinement time of $\tau_{\mathrm{E}}=W / P_{\text {net }} \propto P_{\text {net }}^{-1.0}$. This is shown in figure 14 where the difference between hydrogen and deuterium still can be described with the mass dependence in the confinement time scaling. However, for the discharge with direct ion heating by NBI $\tau_{\mathrm{E}}$ lies directly in between the deuterium and hydrogen point.

To understand these differences it is illustrating to consider electron and ion energy confinement times separately. In the standard 0D approach of the global energy confinement time this is not 

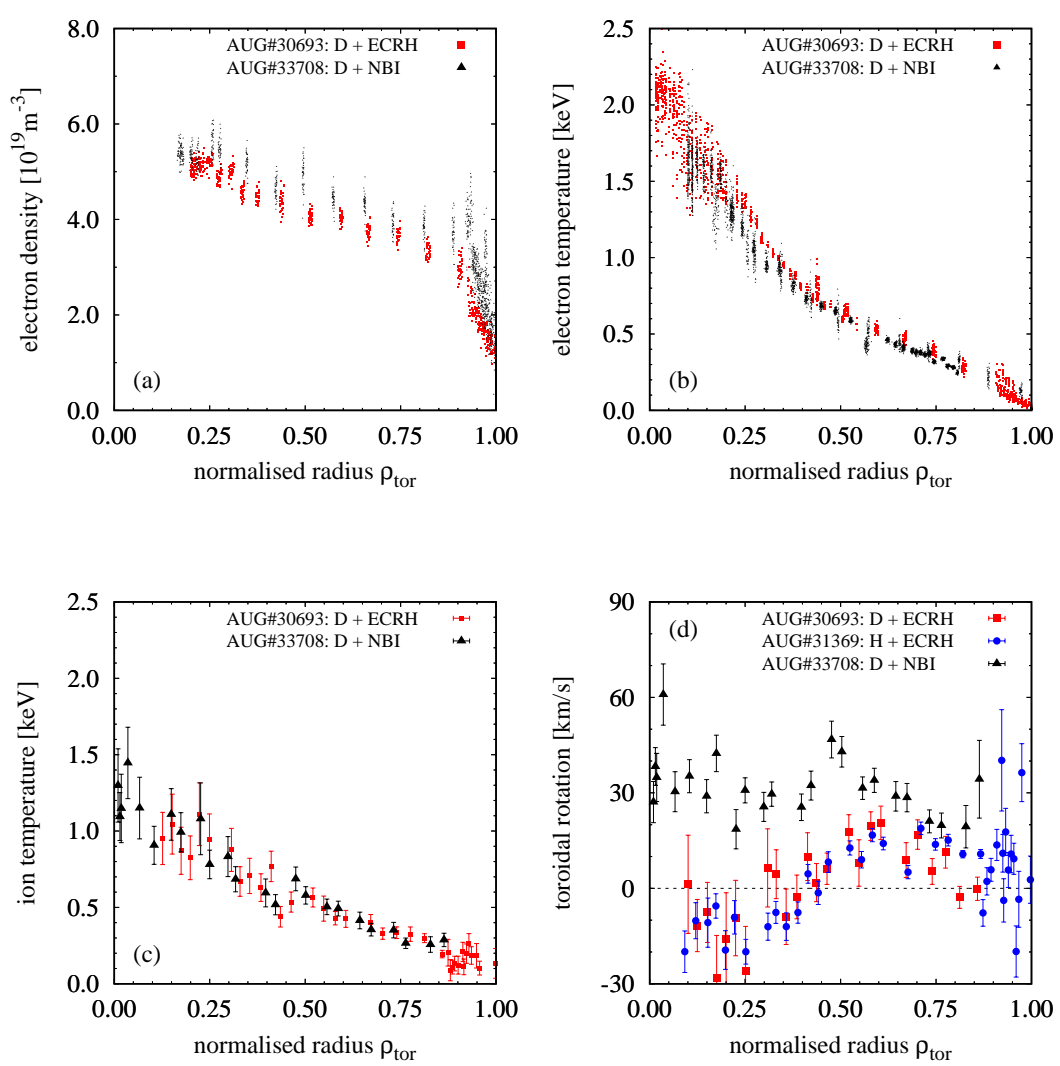

FIG. 13: Radial profiles of electron density (a), electron temperature (b), ion temperature (c) and toroidal rotation (d) for the discharge only ECRH (red) and the one with dominantly NBI heating (black).

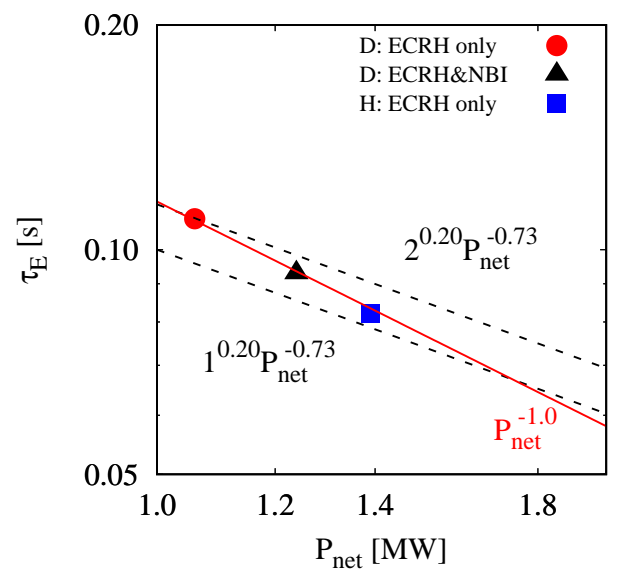

FIG. 14: Global energy confinement time plotted against the net heating power. The lines correspond to different scaling expressions.

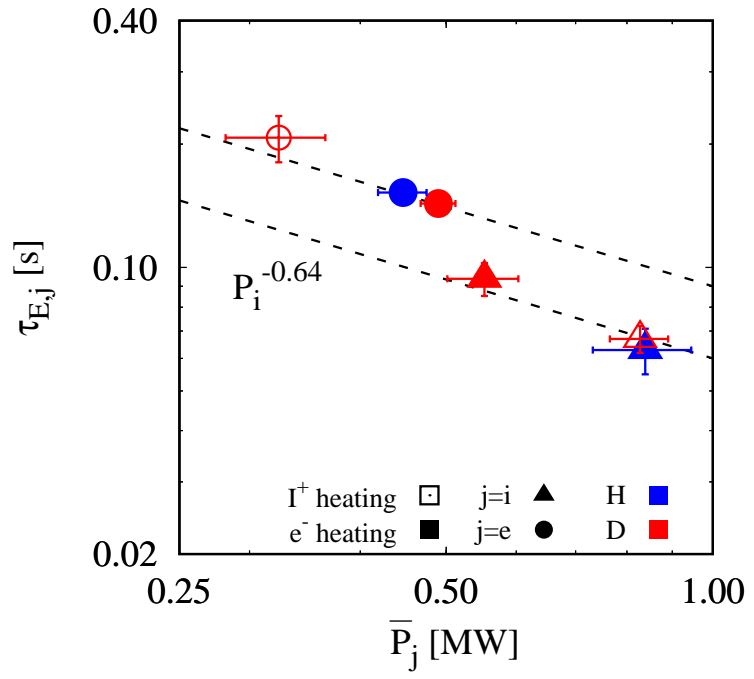

FIG. 15: Thermal energy confinement time plotted vs. heating power and separated in electron and ion channel. The data is from the discharges shown in figure 1 and 12 , filled symbols correspond to only electron heating, open symbols include direct ion heating, circles show the electron and triangles the ion channel, red points correspond to deuterium and blue to hydrogen. The dashed line shows the power degradation for the ion channel derived from the GENE $R / L_{T \mathrm{i}}$ scan. 

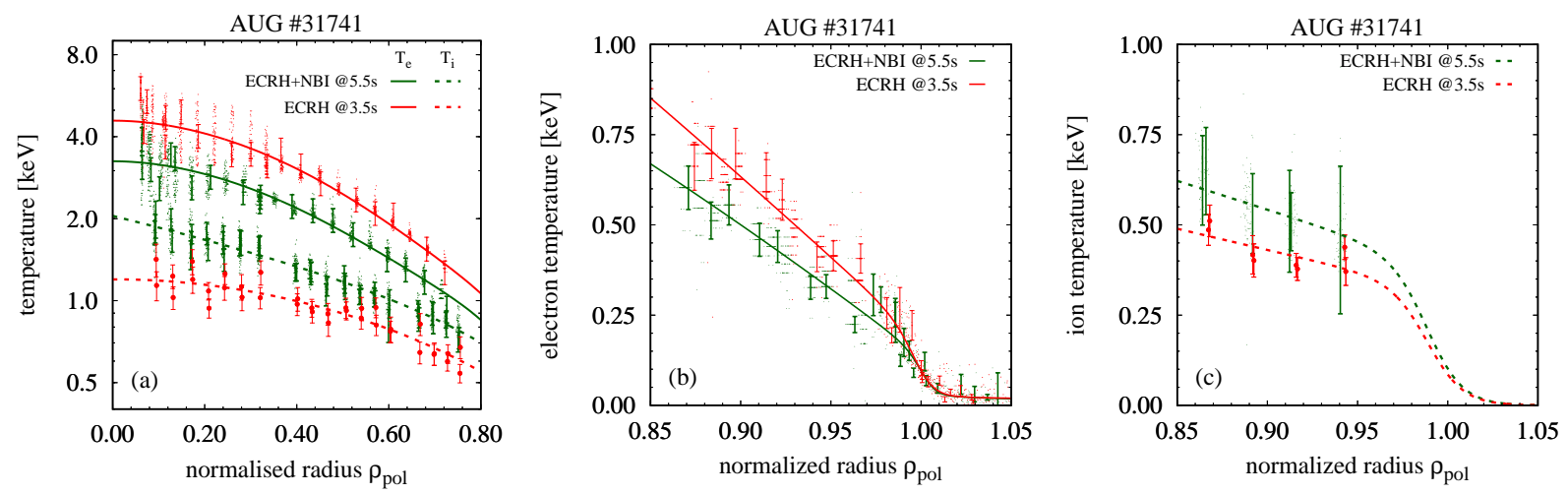

FIG. 16: Temperature profiles for a hydrogen plasma with an electron heated phase (ECRH only) and a phase with similar total power but significant ion heating $(E C R H+N B I)$.

foreseen. In a separate treatment of electrons and ions it is important to take 1D effects into account. This is because the radial profiles of heat sources and sinks can differ significantly for electrons and ions depending on the applied heating method. Central heating is obviously more efficient than off-axis heating. Therefore, an effective averaged heating is defined for each species $\mathrm{j}=\mathrm{e}, \mathrm{i}$ as

$$
\bar{P}_{\mathrm{j}}=\frac{\int \mathrm{d} r Q_{\mathrm{j}}}{\int \mathrm{d} r\left(Q_{\mathrm{e}}+Q_{\mathrm{i}}\right)} P_{\mathrm{net}}
$$

where the renormalisation to $P_{\text {net }}$ is done so that the new effective quantities still compare to the global ones. The species energy confinement time is then $\tau_{\mathrm{E}, \mathrm{j}}=W_{\mathrm{th}, \mathrm{j}} / \bar{P}_{\mathrm{j}}$ with $W_{\mathrm{th}, \mathrm{j}}$ being the thermal stored energy of electrons or ions. The integral is evaluated for the main transport region from the sawtooth inversion radius up to $\rho_{\text {tor }}=0.80$. A variation of the integral limits is taken into account in the uncertainties. With $r_{\mathrm{ei}}=W_{\mathrm{th}, \mathrm{e}} / W_{\mathrm{th}, \mathrm{i}}$ the relation to the global energy confinement time becomes

$$
\tau_{\mathrm{E}}=\frac{\left(1+r_{\mathrm{ei}}\right) \tau_{\mathrm{E}, \mathrm{e}} \tau_{\mathrm{E}, \mathrm{i}}}{\tau_{\mathrm{E}, \mathrm{e}}+r_{\mathrm{ei}} \tau_{\mathrm{E}, \mathrm{i}}} .
$$

When plotting $\tau_{\mathrm{E}, \mathrm{j}}$ for all three discharges in figure 15 it becomes evident that the ion energy confinement time is lower than the electron confinement time for similar heating powers. Additionally, the ion confinement time is the same for hydrogen and deuterium when the same amount of heat is put into the ions. The power degradation expected for the ions from the Gene simulations described in section 2.3 is compatible with the experimental data independently of the main ion mass. The different global energy confinement times arise from the offset between $\tau_{\mathrm{E}, \mathrm{e}}$ and $\tau_{\mathrm{E}, \mathrm{i}}$, it follows for the analysed ITG dominated regime that more heat in the ions or an increase of $\bar{P}_{\mathrm{i}} / \bar{P}_{\mathrm{e}}$ will cause a lower global energy confinement time simply because $\chi_{\mathrm{i}}>\chi_{\mathrm{e}}$.

The density in the three discussed discharges is relatively high and therefore, the coupling of electron and ion heat channels is quite efficient. To illustrate the concept described in the previous paragraph more distinctly, also a low density L-mode is discussed for the remainder of this section. The low density allows more control over the heat ratio because $p_{\mathrm{ei}}$ is negligible in this particular case. The L-mode with hydrogen main ions has two phases with the same total net heating power and achieves for ECRH only heating $\bar{P}_{\mathrm{i}} / \bar{P}_{\mathrm{e}} \sim 0.3$ and with ECRH + NBI heating $\bar{P}_{\mathrm{i}} / \bar{P}_{\mathrm{e}} \sim 0.8$. The resulting profiles are shown in figure 16 (a) where the $T_{\mathrm{e}} / T_{\mathrm{i}}$ ratio decreases significantly with NBI 


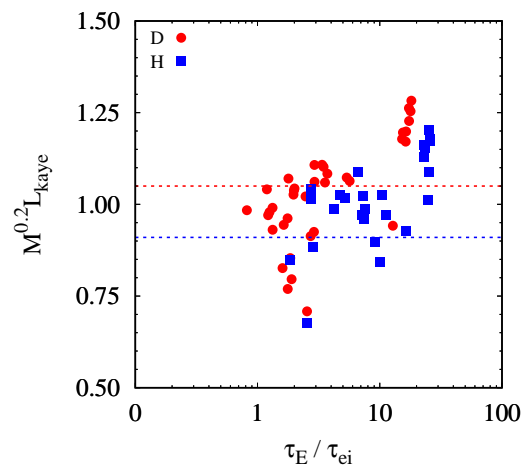

FIG. 17: The L-mode confinement factor without its mass dependence plotted against the ratio of thermal energy confinement time and energy exchange time between electrons and ions. The mass dependence from the scaling is indicated by the dashed lines for hydrogen isotopes $2^{0.2}=1.15$.

heating. The temperature is plotted on a logarithmic scale to highlight the gradient lengths which do not change for the different heating mixes, additionally, $R / L_{T \mathrm{e}}>R / L_{T \mathrm{i}}$ for $\rho_{\mathrm{pol}} \in[0.2,0.8]$ at mid radius $R / L_{T \mathrm{e}}=9.0$ and $R / L_{T \mathrm{i}}=5.0$. The variation in temperature is dominated by the edge as illustrated for the electrons in (b) and for the ions in (c). Despite similar total heating power a difference in confinement is observed: $L_{\mathrm{ECRH}} / L_{\mathrm{ECRH}+\mathrm{NBI}}=1.16$.

To achieve a stored energy match without varying the heating mix - like in the isotope experiments - the power degradation or profile stiffness has to be taken into account. Using again the L-mode scaling law the necessary power becomes $P_{\mathrm{ECRH}+\mathrm{NBI}}=1.7 P_{\mathrm{ECRH}}$. In practise, the application of this amount of heating will likely put the plasma beyond the L to $\mathrm{H}$ threshold, thereby preventing a comparison of L-mode confinement.

A similar discharge run in deuterium confirmed the observations made in hydrogen. In a plasma with different gradient lengths in electron and ion temperature a shift of heat into the channel with lower gradient length will reduce the confinement. This correlation is expected to break with a change of transport parameters induced by the different heating schemes. The correlation is most pronounced in discharges in hydrogen or deuterium when electron and ion channels are decoupled. It should be noted that the observation is consistent with the gyro-Bohm picture for mono-isotopic studies. The heat fluxes vary with temperature as $q / T^{5 / 2}$ for both electrons and ions.

\section{L-mode data set}

The dedicated L-mode discharges presented in the previous section draws a physics picture which should be compatible with a broader database of AUG discharges. For this purpose we analyse the L-mode phase from a L-H transition study in H and D [11. These additional discharges are all with 1.0 MA and -2.5 T, the heating is dominantly ECRH, but includes also phases with NBI particularly in hydrogen. The range of volume averaged effective collisionality for these discharges is 0.003 to 0.070 where upper end corresponds to the discharges discussed in the previous sections. To compare this data set with the $0.8 \mathrm{MA}$ discharges from the $\rho_{\star}$-scan the confinement time is normalized to the L-mode confinement time scaling [6] without its mass dependence of $M^{0.2}$. A parameter to encompass the findings of the previous sections is $\tau_{\mathrm{E}} / \tau_{\mathrm{ei}}$ where $\tau_{\mathrm{ei}}=\tau_{\mathrm{e}} m_{\mathrm{i}} /\left(2 m_{\mathrm{e}}\right)$ is 
the energy exchange time between electrons and ions with the electron-ion collision time $\tau_{\mathrm{e}}$. This ratio depends mainly on isotope mass number and plasma density. In figure 17 the confinement factor is plotted against $\tau_{\mathrm{E}} / \tau_{\mathrm{ei}}$. The separation due to the isotope mass expected from the empirical scaling is indicated by horizontal lines. However, the experimental data does not follow this trend. At a $\tau_{\mathrm{E}} / \tau_{\mathrm{ei}}$ where data for both isotopes exists no separation in confinement time is observed.

This larger data set of AUG L-modes does not support the mass dependence expected from the scaling law, but confirms the results of the dimensionless $\rho_{\star}$ scan: for matched conditions no isotope dependence on confinement is observed.

\section{Discussion}

In ASDEX Upgrade experiments are performed to explore the impact of $\rho_{\star}$ and the ion mass on the core confinement. To analyse core transport effects the impact of the edge plasma is minimized. In particular, ELMs are avoided by choosing an L-mode scenario.

The dimensionless mass- and $\rho_{\star}$-scan reveals that the lower confinement in hydrogen compared to deuterium plasmas is accompanied by a larger ion heat flux due to the enhanced electron ion heat transfer. The electron transport channel is unaffected by the different main ion mass, as indicated by both power balance and perturbative heat transport analysis. In this case the reason for $T_{\mathrm{e}}^{\mathrm{H}}=T_{\mathrm{e}}^{\mathrm{D}}$ is trivially $q_{\mathrm{e}}^{\mathrm{H}}=q_{\mathrm{e}}^{\mathrm{D}}$. A significant impact on the electron temperature in these ITG dominated discharges is also not expected from nonlinear GENE simulations. It remains that the ion heat flux is over $50 \%$ larger in hydrogen compared to deuterium for the same stored energy. The GEnE simulations highlight the importance of the ion heat transport channel. An increase of heat transport due to the isotope mass is not expected for ITG dominated plasmas. It is possible to explain the heat fluxes between simulation and experiments within the uncertainties. However, the ITG turbulence is so sensitive to $R / L_{T i}$ that the ion heat flux can increase significantly while the change in the ion temperature profile remains marginal. When quantifying the sensitivity of $Q_{\mathrm{i}}$ to changes of $R / L_{T \mathrm{i}}$ - or the stiffness of heat transport by GENE - the value is well within the range expected from empirical scaling laws. A reduced stiffness is not compatible with experimental findings. This observation is independent of the isotope mass.

The knowledge from gyrokinetic simulations is used in the ASTRA transport code. The resulting simulations show that it is sufficient to use the same coefficients in the transport model for hydrogen and deuterium and still reproduce the experimental profiles and global confinement properties. Only the well known mass dependence of the electron-ion energy exchange has to be taken into account.

This concept is confirmed by additional experiments with the same isotope mass. They show that larger ion heat fluxes - due to direct ion heating with NBI - will lead to reduced confinement when $R / L_{T \mathrm{i}}<R / L_{T \mathrm{e}}$ and when the type of transport is not changed by the different heating. The different toroidal rotation in the case with direct ion heating by neutral beams could also have an influence on the transport, however, in the core faster rotation is typically observed to stabilise transport [51] and not to destabilise it. Therefore, the change in rotation is unlikely to cause the 
observed reduction in confinement.

In the analysed set of L-mode discharges the appearance or magnitude of the "isotope effect" depends on the electron-ion energy exchange or the applied heating method and less so on a change of transport properties due to a different isotope mass number. However, in H-mode the isotope effect is observed to appear very robustly under all conditions. ELMs and edge stability seem to play a significant part in the understanding of the isotope effect in H-mode [52]. Still it is not the complete picture since already at the $\mathrm{L}-\mathrm{H}$ transition different heat fluxes in the ions are observed when comparing different isotopes [11]. It was proposed that the radial electric field $E_{\mathrm{r}}$ well is the governing parameter and to achieve similar $\nabla T_{\mathrm{i}}$ a higher $q_{\mathrm{i}}$ is necessary in hydrogen. This is consistent with the observation that for direct ion heating with NBI more $q_{\mathrm{i}}$ is required to counteract the reduction of $E_{\mathrm{r}}$ due to faster edge toroidal rotation [53]. In normal L-mode the edge electric field is up to a factor of two below the value at the $\mathrm{L}-\mathrm{H}$ transition [54,55]. The reason for the initial buildup of the $E_{\mathrm{r}}$ is often linked with GAM activity whose amplitude increases towards the L-H transition in low density discharges [56]. The GAMs itself are again influenced by the main ion mass [20,22]. This could be an explanation for the different $q_{\mathrm{i}}$ at the transition. However, in L-mode - well away from the transition where the GAM amplitude is lower - their impact on transport would be less pronounced and it would not necessarily remain significant.

Recently, an additional effect on core turbulence due to isotope mass was discussed, the stabilising effect of $\mathrm{E} \times \mathrm{B}$ shearing. The stabilisation differs with isotope for an ITER like H-mode plasma as predicted by GENE [57]. The $\mathrm{E} \times \mathrm{B}$ effects are also taken into account in the simulations discussed in section 2.3, but due to the much lower plasma rotation their impact on the heat fluxes is negligible. Therefore, the stabilisation of core turbulence by $\mathrm{E} \times \mathrm{B}$ shear is another promising piece of the puzzle to explain the different magnitude of the isotope effect in L- and H-mode.

Altogether, there are a variety of effects that help to understand the isotope dependence of confinement in H-mode, however, all these effects are expected to become smaller if not negligible in L-mode. Therefore, it is not surprising to observe a weaker or no isotope dependence of heat transport in L-mode.

It was pointed out that the ratio of ion to electron heat - which is influenced by the isotope mass - can cause a change in the global energy confinement time without the necessity of an isotope dependence in the heat transport. In such a situation $\tau_{\mathrm{E}}$ is not suited to describe the underlying transport physics.

Due to the mass dependence in the collisional energy exchange term the confinement depends crucially on the properties of the underlying transport mechanism. In particular, the different stiffness values of the electron and ion heat transport become important. In the ITG dominated regime discussed here the higher stiffness of the ion heat transport leads to a reduced confinement in hydrogen. In contrast to that, recently studied JET L-mode plasmas exhibited a significant ETG contribution to the transport resulting in a higher stiffness of the electron heat transport and $\chi_{\mathrm{s}, \mathrm{e}}>\chi_{\mathrm{s}, \mathrm{i}}[58]$. This might lead to a different isotope dependence of confinement.

The difference in $\tau_{\mathrm{E}}$ can be explained with the electron and ion energy exchange. However, a small impact of the isotope mass on the transport coefficients cannot be excluded within the 
measurement uncertainties. This is found for an ITG dominated case and should be investigated in different turbulence regimes e.g. ETG or TEM dominated plasmas.

In terms of future devices and tritium operation the findings of this paper suggest only a small or negligible isotope dependence of transport in L-mode. Due to larger machine size and longer energy confinement times the impact of the isotope mass on the energy equilibration time will become less important than for the medium sized electron heated plasmas investigated here. This will result in a reduced impact of differences in electron and ion heat transport when comparing plasmas with different isotopes. However, the L-mode results are not applicable to H-mode where the pedestal and the ELMs seem to govern the isotope effects.

The authors want to thank C. Angioni, H. Meyer, E. Solano and U. Stroth for helpful discussions. The simulations presented in this work were carried out using the HELIOS supercomputer system at Computational Simulation Centre of International Fusion Energy Research Centre (IFERCCSC), Aomori, Japan, and the HYDRA supercomputer at the Rechenzentrum Garching (RZG), Germany. GENE linear runs have been carried out in Uranus, the local HPC cluster located at Universidad Carlos III de Madrid (Spain) funded by EU FEDER funds and by the National Projects UNC313-4E-2361, ENE2009-12213-C03-03, ENE2012-33219 and ENE2012-31753. This work has been carried out within the framework of the EUROfusion Consortium and has received funding from the Euratom research and training programme 2014-2018 under grant agreement No 633053. The views and opinions expressed herein do not necessarily reflect those of the European Commission.

[1] Wootton, A. J. et al., Physics of Fluids B 2 (1990).

[2] PETTY, C. C., Physics of Plasmas 15 (2008) 80501.

[3] WALTZ, R. E. et al., Physical Review Letters 65 (1990) 2390.

[4] BeSSENRODT-WEBERPALS, M. et al., Nuclear Fusion 33 (1993) 1205.

[5] ITER Physics Expert Group, Nuclear Fusion 39 (1999) 2137.

[6] KAYE, S. M. et al., Nuclear Fusion 37 (1997) 1303.

[7] SCHISSEL, D. P. et al., Nuclear Fusion 29 (1989) 185.

[8] URANO, H. et al., Nuclear Fusion 56 (2016) 16005.

[9] SCHNEIDER, P. A. et al., Plasma Physics and Controlled Fusion 57 (2015) 14029.

[10] CORDEY, J., Nuclear Fusion 39 (1999) 1763.

[11] RYTER, F. et al., Plasma Physics and Controlled Fusion 58 (2016) 14007.

[12] LAGGneR, F. M. et al., Physics of Plasmas 24 (2017) 56105.

[13] STAMBAUGH, R. et al., Plasma Physics and Controlled Fusion 30 (1988) 1585.

[14] MURMAnN, H. et al., The Isotope Dependence of Global Confinement in Ohmically and Auxiliary Heated ASDEX Plasmas, in 15th European Conference on Controlled Fusion and Plasma Heating, edited by S. Pesic, J. J., Dubrovnik, 1988.

[15] STROTH, U. et al., Nuclear Fusion 36 (1996) 1063.

[16] STROTH, U. et al., Physica Scripta 51 (1995) 655.

[17] ITOH, K. et al., Physics of Plasmas 13 (2006).

[18] HAHM, T. S. et al., Nuclear Fusion 53 (2013) 72002.

[19] XU, Y. et al., Phys. Rev. Lett. 110 (2013) 265005. 
[20] GURChenkO, A. D. et al., Plasma Physics and Controlled Fusion 58 (2016) 44002.

[21] LIU, B. et al., Nuclear Fusion 56 (2016) 56012.

[22] HENNEQUIN, P. et al., Comprehensive experimental study of plasma turbulence structure and its scaling with $\rho_{\star}$, in 42th EPS Conference on Plasma Physics, page I1.102, Lisbon, 2015.

[23] BUSTOS, A. et al., Physics of Plasmas 22 (2015) .

[24] IDOMURA, Y. et al., Physics of Plasmas 21 (2014) .

[25] DIF-PRADAliER, G. et al., Phys. Rev. Lett. 114 (2015) 85004.

[26] WAGNER, D. et al., Nuclear Fusion 48 (2008) 54006.

[27] KURZAN, B. et al., Review of Scientific Instruments 82 (2011) 103501.

[28] WOLFRUM, E. et al., Review of Scientific Instruments 64 (1993) 2285.

[29] SALMON, A. N., International Journal of Infrared and Millimeter Waves 15 (1994) 53.

[30] PUETTERICH, T. et al., Fast CXRS-Measurements in the Edge Transport Barrier of ASDEX Upgrade, in 35th EPS Conference on Plasma Phys. Hersonissos, volume 32, pages $\mathrm{P}-2.083,2008$.

[31] VIEZZER, E. et al., Review of Scientific Instruments 83 (2012) 103501.

[32] MCDERmotT, R. M. et al., Nuclear Fusion 54 (2014) 43009.

[33] RATHGEBER, S. K. et al., Plasma Physics and Controlled Fusion 52 (2010) 95008.

[34] BERNERT, M. et al., Review of Scientific Instruments 85 (2014) .

[35] DUNNE, M. G. et al., Nuclear Fusion 52 (2012) 123014.

[36] HAWRYLUK, R. J., An Empirical Approach to Tokamak Transport, in Phys. Plasmas Close Thermonucl. Cond., volume 1, page 19, 1980.

[37] PORCELLI, F. et al., Plasma Physics and Controlled Fusion 38 (1996) 2163.

[38] WeSSON, J. A., Tokamaks, Clarendon Press, Oxford, second edition, 1997.

[39] IMBEAUX, F. et al., Plasma Physics and Controlled Fusion 43 (2001) 1503.

[40] RYTER, F. et al., Nuclear Fusion 43 (2003) 1396.

[41] LOPES-CARDOZO, N. J. et al., Plasma Physics and Controlled Fusion 32 (1990) 983.

[42] JACCHIA, A. et al., Physics of Fluids B 3 (1991).

[43] JENKO, F. et al., Physics of Plasmas 7 (2000) 1904.

[44] XANThopoulos, P. et al., Physics of Plasmas 16 (2009).

[45] PEREVERZEV, G. V. et al., IPP Report 5/98 (2002).

[46] Garbet, X. et al., Plasma Physics and Controlled Fusion 46 (2004) 1351.

[47] RYTER, F. et al., Plasma Physics and Controlled Fusion 48 (2006) B453.

[48] ASP, E. et al., Plasma Physics and Controlled Fusion 49 (2007) 1221. 
[49] MANINI, A. et al., Nuclear Fusion 46 (2006) 1047.

[50] MLYNEK, A. et al., Nuclear Fusion 51 (2011) 43002.

[51] MANTICA, P. et al., Phys. Rev. Lett. 102 (2009) 175002.

[52] URANO, H. et al., Nuclear Fusion 53 (2013) 83003.

[53] RYTER, F. et al., Nuclear Fusion 54 (2014) 83003.

[54] Groebner, R. J. et al., Phys. Rev. Lett. 64 (1990) 3015.

[55] VIEZZER, E. et al., Plasma Physics and Controlled Fusion 56 (2014) 75018.

[56] CONWAY, G. D. et al., Phys. Rev. Lett. 106 (2011) 65001.

[57] GARCIA, J. et al., Plasma Physics and Controlled Fusion 59 (2017) 14023.

[58] BONANOMI, N. et al., Electron Heat Transport in JET from Ion to Electron scales:

Experimental Investigation and Gyro-kinetic Simulations, in 25th IAEA Fusion Energy Conference, pages EX/P6-14, Kyoto, 2016, IAEA, Vienna. 